\title{
Histological examination of peroperative frozen sections in suspected lung cancer
}

\author{
S A M Nashef, J G Kakadellis, P S Hasleton, J S Whittaker, C M Gregory, M T Jones
}

\begin{abstract}
Background-Despite extensive investigations some patients with suspected lung cancer may undergo thoracotomy without preoperative histological proof of malignancy. A questionnaire on the use of histological examination of peroperative frozen sections in such patients was sent to 50 thoracic surgeons. Replies were received from 41 surgeons and indicated an absence of consensus on the usefulness of histological examination of frozen sections in this context, confirm-
\end{abstract} ing the need for this study.

Methods-During one year 60 consecutive patients undergoing thoracotomy for suspected lung cancer without a prior histological diagnosis were studied prospectively. At thoracotomy the surgeon assessed the lesion macroscopically and a verdict on whether it was malignant was recorded. A biopsy specimen was then taken for examination of a frozen section and the result recorded. The appropriate operation was performed and the surgeon's verdict and the report on the frozen section were compared with the definitive histological diagnosis based on a paraffin section.

Results-Of 50 malignant lesions, 43 were identified by the surgeon and 47 by examination of the frozen section (sensitivity $86 \%$ and $94 \%$ respectively). Of 10 benign lesions, four were identified by the surgeon and nine by examination of the frozen section (specificity $40 \%$ and $90 \%$ respectively).

Conclusions-Clinical and macroscopic assessment at thoracotomy are inferior to examination of frozen sections in suspected lung cancer, particularly where the lesion is benign. Lung resection should not be performed without examination of peroperative frozen sections when thoracotomy is performed for suspected but unproved lung cancer.

(Thorax 1993;48:388-389)

Most patients undergoing thoracotomy for lung cancer have a histologically confirmed diagnosis before operation. Some, however, come to thoracotomy without a definitive preoperative histological diagnosis despite all the investigations they have had. Few surgeons would contemplate mastectomy without histological or cytological confirmation of malignancy. Thoracotomy for suspected lung cancer, however, is sometimes apparently performed without such confirmation: discussions at a recent national thoracic surgical meeting of young consultant cardiothoracic surgeons (Leeds, 1990) indicated a wide variation in perception of the usefulness of frozen section histology (FS) in this context, which prompted this study.

\section{Methods}

\section{SURVEY}

A questionnaire was sent to 50 consultant thoracic surgeons from all regions of the United Kingdom. The respondents were asked to choose from five options the one which best represented their practice when they were performing thoracotomy for suspected lung cancer with no definitive preoperative histological diagnosis. The options were:

A: I would proceed to lung resection on the basis of the clinical picture and macroscopic features of the lesion. I do not rely on FS in these cases.

B: I would proceed to lung resection on the basis of the clinical picture and macroscopic features of the lesion. I may perform FS if the clinical picture or the macroscopic appearances are atypical.

C: I would perform lobectomy (or wedge resection) on the basis of the clinical picture and macroscopic features of the lesion, but I would perform FS before proceeding to pneumonectomy.

D: I would not perform lobectomy or pneumonectomy without histological diagnosis.

E: None of the above. My view is

There were 41 respondents (response rate $82 \%$ ), of whom one chose option A (never use FS), 17 option B (FS in atypical cases), 11 option C (FS before pneumonectomy) and eight option D (FS always). Four surgeons expressed other views (option E).

\section{Patients and methods}

During one year 60 patients ( 33 male) underwent thoracotomy at Wythenshawe Hospital for suspected lung cancer with no preopera- 
tive histological diagnosis and all were entered into the study.

All operations were carried out in the thoracic operating theatre, which is located at the opposite end of the hospital from the histopathology laboratory (five minutes' brisk walk). Once the chest was opened the surgeon was asked by the circulating nurse to decide whether he thought the lesion was cancer. An appropriate biopsy was then performed and the specimen carried to the laboratory by the theatre porter. The tissue was frozen with liquid nitrogen and $5 \mathrm{~mm}$ sections were cut, stained with haematoxylin and eosin, and examined by one of two consultant histopathologists (PSH and JSW). The appropriate operation was carried out when the report was telephoned to the surgeon. A definitive histological examination of paraffin sections was performed in all cases on the frozen section material as well as on any subsequently excised lung tissue. The following information was recorded for all patients: date of operation, surgeon's opinion, time of biopsy, time of telephoned report on the frozen section, nature of the lesion according to the report on the frozen section, operation performed, and definitive histological report.

\section{Results}

The 60 patients studied represented $13 \%$ of the patients having major thoracic surgery ( $n=453$ ) and $26 \%$ of those having thoracotomies for lung resection ( $n=229$ patients) at the Wythenshawe Hospital in one year.

The mean time from biopsy to the telephoned report on the frozen section was 27 minutes (range 15-50 minutes).

Of the 60 patients, $50(83 \%)$ were found to have lung cancer according to the definitive histological examination and the remaining 10 had benign lesions (focal pulmonary fibrosis 6, tuberculosis 2, adenochondroma 2). Lung resection was performed in 54 patients: pneumonectomy in nine, lobectomy in 33, and wedge resection in 12 . No further resection was considered necessary in the remaining six patients, all of whom had benign lesions.

Of the 50 malignant lesions, 43 were identified by the surgeon and 47 by frozen section examination (sensitivity $86 \%$ and $94 \%$ ). Of the 10 benign lesions, four were identified by the surgeons and nine by frozen section examination (specificity $40 \%$ and $90 \%$ ). In the 10th patient there was insufficient tissue on the frozen section for a diagnosis to be made; the lesion was not reported as malignant.

There were no differences in the histological diagnosis made by frozen section examination and paraffin section examination of the same biopsy specimens. Where the frozen section report differed from the definitive diagnosis this was invariably due to sampling.

\section{Discussion}

Our survey confirms that there is a lack of consensus among thoracic surgeons in the United Kingdom about the value and indications of intraoperative frozen section examination in suspected lung cancer. Only eight of 41 surgeons said that they would use frozen section examination systematically before contemplating lung resection. In the absence of histological diagnosis the only guide to the nature of the lesion is the macroscopic assessment by the surgeon. Our study indicates that this is inferior to frozen section examination both in sensitivity and in specificity. That this difference appears to be more pronounced in benign lesions is particularly worrying as it raises the possibility that pneumonectomy is performed for benign disease, with serious functional and medicolegal sequelae.

Most patients undergoing thoracotomy for lung cancer have a well established preoperative histological diagnosis $(74 \%$ in our hospital). The proportion that do not may vary between institutions, depending on the zeal with which such a diagnosis is pursued. This proportion could perhaps be reduced by systematic use of investigations such as needle biopsy under computed tomography or other guidance, thoracoscopy, or limited diagnostic thoracotomy. These investigations are, however, costly and not without risk, and they further delay operation in a subgroup of patients in whom the most likely diagnosis remains lung cancer and who should therefore undergo resection with the minimum of delay. Furthermore, these investigations are useful only if they confirm malignancy: reported absence of malignancy may be due to sampling error and is therefore unlikely to provide the basis on which thoracotomy may be avoided.

Even with the most ambitious diagnostic efforts some patients with suspected lung cancer will come to thoracotomy without preoperative histological confirmation and a proportion of these patients will have benign disease. It is vitally important that these patients are identified to avoid needless and possibly disabling lung resection. Our study indicates that systematic use of frozen section is superior to macroscopic evaluation in identifying these patients. As a result of this study we now believe that no patient should undergo lung resection without histological diagnosis.

We thank Mr H Moussalli, Mr R A M Lawson, Mr A K Deiraniya, Mr A N Rahman, and Mr C S Campbell for allowing us to study their patients. 